\title{
Simulação do processo de destilação extrativa para produção de etanol anidro utilizando etilenoglicol como agente de separação
}

\author{
Simulation of the extractive distillation process to produce anhydrous ethanol using ethylene glycol
} as a separating agent

Simulación del proceso de destilación extractiva para la producción de etanol anhidro utilizando etilenglicol como separador

\author{
Alvaro Eduardo Costa Souza \\ ORCID: https://orcid.org/0000-0002-7209-0135 \\ Universidade Federal do Triângulo Mineiro, Brasil \\ E-mail: alvarocosta2097@gmail.com \\ Daniel Alves Cerqueira \\ ORCID: https://orcid.org/0000-0003-3195-1995 \\ Universidade Federal do Triângulo Mineiro, Brasil \\ E-mail: daniel.cerqueira@uftm.edu.br \\ Nádia Guimarães Sousa \\ ORCID: https://orcid.org/0000-0002-3142-5875 \\ Universidade Federal do Triângulo Mineiro, Brasil \\ E-mail: nadia.sousa@uftm.edu.br \\ Cássia Regina Cardoso \\ ORCID: https://orcid.org/0000-0002-3226-7653 \\ Universidade Federal do Triângulo Mineiro, Brasil \\ E-mail: cassia.cardoso@uftm.edu.br
}

\begin{abstract}
Resumo
O etanol anidro é um produto com teor mínimo de 99,5\% molar de etanol, possui aplicações como solvente, precursor de compostos químicos, além de poder ser adicionado à gasolina para uso como combustível. A separação da mistura etanol/água por destilação fracionada é limitada a $88 \%$ molar de etanol devido à formação de um azeótropo, o que impede a produção de etanol anidro. Métodos alternativos de destilação são utilizados, destacando-se a destilação extrativa, que consiste na introdução de um agente extrator de alto ponto de ebulição, capaz de absorver e realizar o arraste da água para a base da coluna, o etanol anidro é obtido como destilado. Este trabalho propõe uma simulação no software livre ChemSep do processo de destilação extrativa para obtenção de etanol anidro utilizando etilenoglicol como agente extrator para eficiência dos estágios de $70 \%$ e $100 \%$. Foram realizadas análises de parâmetros do processo como o número de estágios totais, estágio de alimentação da mistura azeotrópica e do solvente, razão de refluxo e temperatura de alimentação do solvente e da mistura azeotrópica; buscando encontrar as condições de maior eficiência. Como resultado, para eficiência de 100\%, obteve-se uma coluna com 20 estágio totais, com a mistura azeotrópica sendo introduzida no estágio 10 e o solvente no estágio 3, nas temperaturas de $25^{\circ} \mathrm{C}$ e $70^{\circ} \mathrm{C}$ respectivamente, com razão de refluxo de 1,1. Constatou-se a necessidade de um número maior de estágios e de razão de refluxo para obtenção de mesma concentração de etanol no destilado para $70 \%$ de eficiência.
\end{abstract}

Palavras-chave: Destilação extrativa; Simulação; Etanol anidro.

\begin{abstract}
Anhydrous ethanol is a product with a minimum ethanol content of 99,5\% molar, it is used as a solvent, precursor to chemical compounds, and can be mixed to gasoline when used as a fuel. The separation of the ethanol/water mixture by fractional distillation is limited to $88 \%$ molar of ethanol due to the formation of an azeotrope, which hinders the production of anhydrous ethanol. Alternative distillation methods are used, highlighting the extractive distillation, which consists of the introduction of a high boiling point extraction agent, capable of absorbing and dragging the water to the base of the column, anhydrous ethanol is obtained as a distillate. This work proposes a simulation in the free software ChemSep of the extractive distillation process to obtain anhydrous ethanol using ethylene glycol as an extracting agent for $70 \%$ and $100 \%$ stage efficiency. An analysis of the process parameters was performed, such as the number of total stages, feeding stage of the azeotropic mixture and of the solvent, reflux ratio and feeding temperature of the solvent and azeotropic mixture, aiming to find the most efficient conditions. As a result, for $100 \%$
\end{abstract}


Research, Society and Development, v. 10, n. 4, e45610414228, 2021

(CC BY 4.0) | ISSN 2525-3409 | DOI: http://dx.doi.org/10.33448/rsd-v10i4.14228

efficiency, a column with 20 total stages was obtained, with the azeotropic mixture being introduced in stage 10 and the solvent in stage 3 , at temperatures of $25^{\circ} \mathrm{C}$ and $70^{\circ} \mathrm{C}$ respectively, with a reflux ratio of 1.1. A higher number of stages and a higher reflux ratio were necessary to obtain the same concentration of ethanol in the distillate for $70 \%$ stage efficiency.

Keywords: Extractive distillation; Simulation; Anhydrous ethanol.

\section{Resumen}

El etanol anhidro es un producto con un contenido mínimo de etanol del 99,5\% molar, tiene aplicaciones como solvente, precursor de compuestos químicos, además de agregarse a la gasolina para uso en medios de transporte. La separación de la mezcla de etanol/agua por destilación fraccionada está limitada al molar de etanol del 88\% debido a la formación de un azeótropo, que impide la producción de etanol anhidro. Se utilizan métodos alternativos de destilación, destacando la destilación extractiva, que consiste en la introducción de un agente extractor de alto punto de ebullición, capaz de absorber y realizar el arrastre de agua a la base de la columna, etanol anhidro se obtiene como destilado. Este trabajo propone una simulación en software libre de ChemSep del proceso de destilación extractiva para obtención etanol anhidro utilizando etilenglicol como agente extractor para la eficiencia de las etapas de $70 \%$ y $100 \%$. Se realizaron análisis de parámetros del proceso como el número de etapas totales, la etapa de alimentación de la mezcla azeotrópica y del solvente, relación de reflujo y la temperatura de alimentación del solvente y mezcla azeotrópica, con el fin de encontrar las condiciones de mayor eficiencia. Como resultado, para una eficiencia del $100 \%$, se obtuvo una columna con 20 etapas totales, con la mezcla de azeotrópicos que se introdujo en la etapa 10 y el solvente en la etapa 3 , a temperaturas de $25^{\circ} \mathrm{C}$ y $70^{\circ} \mathrm{C}$, respectivamente, con una relación de reflujo de 1,1 . Se encontró la necesidad de un mayor número de etapas y relación de reflujo para obtener la misma concentración de etanol en el destilado para una eficiencia del $70 \%$.

Palabras clave: Destilación extractiva; Simulación; Etanol anhidro.

\section{Introdução}

A destilação fracionada é uma operação de separação ou purificação fundamentada na diferença de volatilidade dos componentes de uma mistura, a fase vapor gerada por evaporação é rica nos componentes mais voláteis enquanto os menos voláteis se concentram na fase líquida. Classifica-se como destilação binária se a mistura submetida à destilação for composta por dois componentes, na presença de mais componentes denomina-se destilação multicomponente (Bessa, 2012, Tadini et al., 2016)

Apesar da possibilidade de ser conduzida em batelada, industrialmente é mais comum a destilação contínua em colunas de estágios com refluxo, onde a mistura é alimentada em um estágio intermediário. A fase líquida desce por diferença de densidade e é parcialmente vaporizada pelo refervedor (fonte de calor), o vapor gerado percorre caminho inverso do líquido. Dessa forma, há contato e transferência de massa entre as fases em cada um dos estágios. O líquido restante é retirado como produto de fundo, já o vapor é condensado e dividido em duas correntes, uma retorna para a coluna (refluxo) e a outra é removida como destilado (Bessa, 2012).

O álcool etílico ou etanol é produto da ação de leveduras sob açúcares, processo intitulado de fermentação. A fermentação ocorre diretamente através de matérias primas sacaríneas, como cana-de-açúcar e beterraba, mas pode também ser efetuada com amiláceas a partir de um tratamento prévio de conversão do amido. Cita-se ainda o álcool proveniente de materiais celulósicos, denominado etanol de segunda geração. A cana-de-açúcar se destaca no território brasileiro devido a maior produtividade de álcool (Muller et al., 2019, Silva et al., 2019).

A produção do etanol começou a se desenvolver no Brasil no início do século XX como uma maneira de desviar o excedente do processamento de açúcar, o etanol gerado era adicionado à gasolina na proporção de 5\% (Leal, 2014). Um marco para a evolução foi a instauração do programa nacional do álcool (Proálcool) em 1975, o intuito era de diminuir a importação de petróleo, o que incentivou o surgimento do veículo totalmente movido a etanol na mesma década (Gimenez et al., 2018). Atualmente, o Brasil está atrás apenas dos Estados Unidos na produção mundial, com geração de 35,6 bilhões de litros de etanol na safra 2019/2020 (CONAB, 2020). Atualmente, diversos países adicionam etanol à gasolina em proporções que 
variam normalmente entre 5\% e 27\%, não apenas para reduzir a utilização de combustíveis fósseis, mas também para aumentar a octanagem da gasolina (Abdellatief et al., 2021).

As usinas de cana-de-açúcar normalmente processam integradamente açúcar e etanol. Como consequência, operações como lavagem da cana e extração do caldo são comuns para ambas as linhas, como mostrado na Figura 1.

Figura 1: Fluxograma da produção de álcool e açúcar.

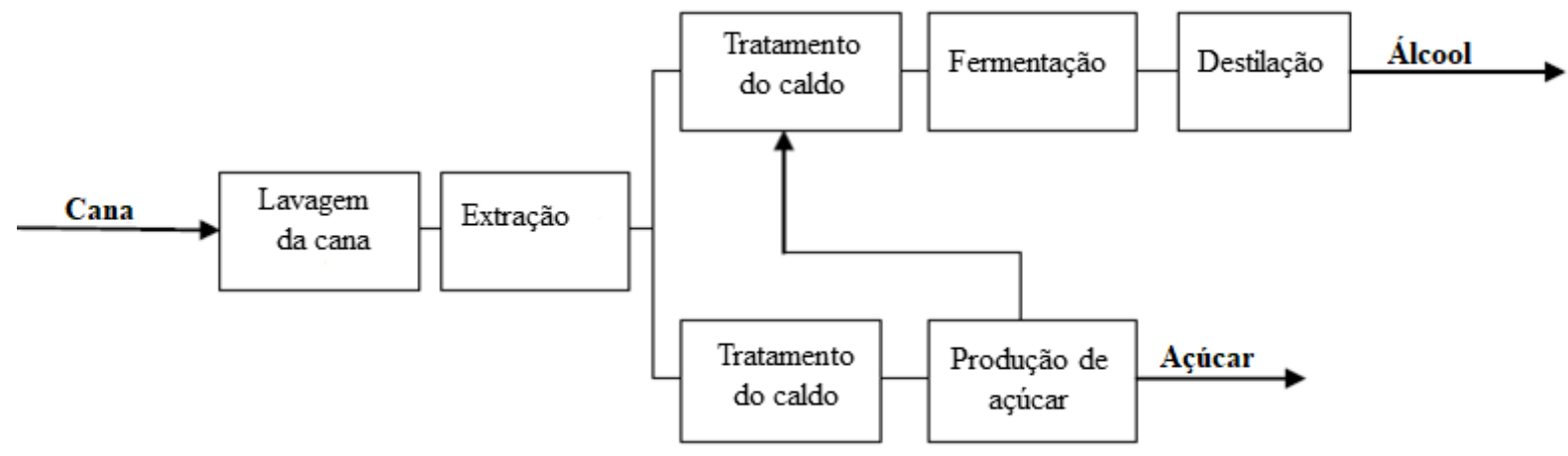

Fonte: Oliveira et al. (2016).

O caldo é extraído por um sistema de moendas e dividido entre as linhas, a porção que segue para processamento de etanol é tratada (para eliminação de impurezas e correção de pH), concentrada e encaminhada para dornas de fermentação. O melaço (subproduto da produção de açúcar) pode ser adicionado ao caldo na etapa de tratamento para aumentar a produção de álcool. O vinho ou mosto (caldo fermentado) é enviado para destilação com 7 a $10 \%$ em massa de álcool etílico (Dias et al., 2015). O produto da destilação é chamado de etanol hidratado, posteriormente este pode ser submetido a purificação para obtenção de etanol anidro ou etanol neutro (Tadini et al., 2016).

A destilação alcoólica é complexa pois o mosto é uma mistura hidroalcóolica constituída de diversos componentes minoritários de classes orgânicas e volatilidades distintas, denominados congêneres, que devem ser eliminados para obtenção de etanol de qualidade. A configuração mais utilizada pelas destilarias está esquematizada na Figura 2.

O mosto fermentado (vinho) alimenta a coluna A' (seção de depuração do vinho) com intuito de eliminar os congêneres mais leves, a fração mais volátil é movida para coluna D e a mais pesada para coluna A (seção de esgotamento do vinho). Na coluna A, a vinhaça, um efluente com alto poder fertilizante, é eliminada como produto de fundo. Praticamente todo etanol exaurido do vinho é transferido através da corrente flegma para a coluna BB1, onde é concentrado até atingir os teores requeridos de etanol. A flegmaça, produto de fundo da coluna BB1, é constituída majoritariamente de água, portanto pode ser descartada sem tratamentos ou utilizada na lavagem da usina (Tadini et al., 2016).

O etanol anidro possui diversas aplicações na indústria, como de matéria prima para produção de ésteres e éteres e como solvente na produção de tintas, medicamentos e alimentos. No Brasil, o etanol é obrigatoriamente adicionado em $27 \%$ à gasolina comum e em 25\% à gasolina premium (PORTARIA $N^{o}$ 75, de 5 de março de 2015 do Ministério da Agricultura, Pecuária e Abastecimento, 2015). O etanol anidro deve possuir no mínimo 99,5\% de etanol em percentual molar (Gil et. al, 2014). 
Figura 2: Esquema da destilação alcoólica.

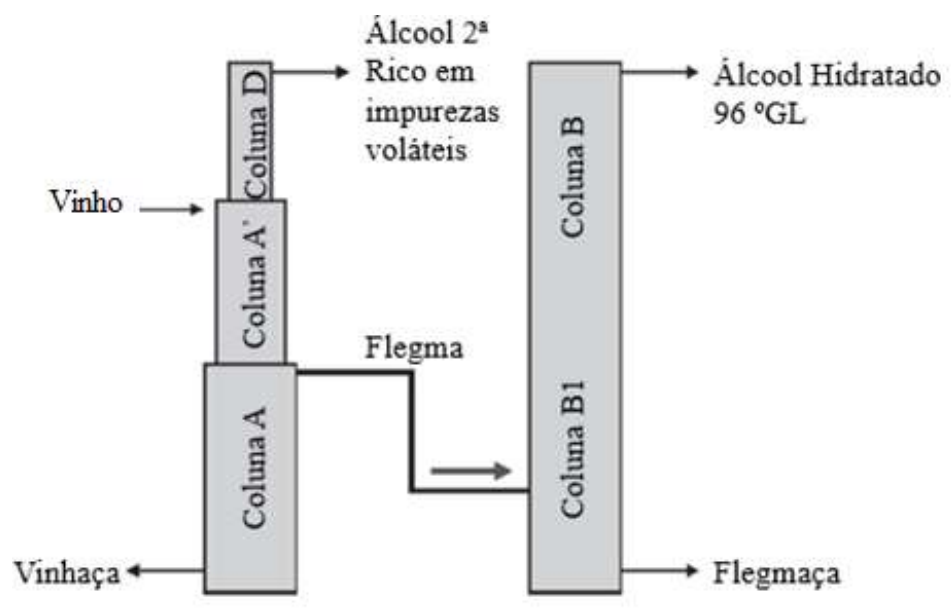

Fonte: Lopes et al. (2011).

Para o estudo da destilação é necessário o conhecimento dos dados de equilíbrio de fases dos componentes, visto que existem certas misturas que em determinada concentração dos constituintes viabilizam a formação de uma mistura com ponto de ebulição constante denominadas misturas azeotrópicas, impossibilitando a separação por destilação fracionada convencional. A interação água/etanol resulta em um azeótropo com teor de etanol de $88,0 \%$ molar a $78,15^{\circ} \mathrm{C}$; o que dificulta a produção de etanol anidro combustível por destilação convencional, já que a concentração de álcool ultrapassa o azeótropo (Cavalcanti, 2018). Métodos alternativos de separação são utilizados pelas indústrias, destacando-se a destilação azeotrópica heterogênea, destilação extrativa, adsorção em peneira molecular e pervaporação (Gil et. al, 2014).

A destilação extrativa é um método baseado na vaporização da mistura com introdução de um solvente seletivo (agente extrator) com ponto de ebulição alto, capaz de interagir diferentemente com cada um dos componentes e alterar a sua volatilidade relativa. Os principais agentes extratores utilizados são: glicerol, glicóis, sais inorgânicos e acetato. Na destilação extrativa para obtenção de etanol anidro, o agente extrator é normalmente alimentado em estágio acima da corrente da mistura água/etanol, realiza a absorção da água e a arrasta para a parte inferior da coluna. A mistura solvente/água é retirada como produto de fundo enquanto o etanol anidro é obtido no topo (Gil, 2014). A Figura 3 mostra uma representação da coluna de destilação extrativa para obtenção de etanol anidro.

A eficiência de uma operação está relacionada com a otimização de alguns parâmetros. No caso da destilação, a temperatura e o estágio de alimentação, a quantidade total de estágios e razão de refluxo são alguns parâmetros importantes, porém devido ao alto custo de operação é inviável a constante variação destes em escala industrial. Uma alternativa é o uso de softwares computacionais para simulação e previsão das respostas do processo, são exemplos de softwares: CHEMCAD, Aspen Plus, ChemSep e HYSYS. O ChemSep é um software que possui uma versão gratuita capaz de simular operações de absorção gasosa, extração líquido-líquido e destilação com mais de 400 componentes disponíveis em sua biblioteca (Santos, 2016). Um apanhado de trabalhos publicados previamente sobre simulação da destilação extrativa está apresentado no quadro 1.

O objetivo deste trabalho foi simular a destilação extrativa para obtenção de etanol anidro combustível utilizando etilenoglicol como agente extrator. Na simulação foram avaliados alguns parâmetros de interesse a fim de obter informação sobre condições de operação eficientes para esse processo. 
Research, Society and Development, v. 10, n. 4, e45610414228, 2021

(CC BY 4.0) | ISSN 2525-3409 | DOI: http://dx.doi.org/10.33448/rsd-v10i4.14228

Quadro 1: Trabalhos publicados anteriormente sobre simulação da destilação extrativa.

\begin{tabular}{|c|c|c|c|c|}
\hline Autores & Objetivo do estudo & Ferramenta de simulação & $\begin{array}{c}\text { Modelo } \\
\text { termodinâmico }\end{array}$ & Agente extrator \\
\hline $\begin{array}{l}\text { Restrepo \& Aguilar- } \\
\quad \text { Arias, } 2003\end{array}$ & $\begin{array}{l}\text { Obtenção de um modelo do } \\
\text { estágio de equilíbrio em estado } \\
\text { estacionário para destilação } \\
\text { extrativa salina utilizando } \\
\text { equações MESH e simulação da } \\
\text { produção de etanol anidro. } \\
\text { Comparação com dados } \\
\text { experimentais }\end{array}$ & $\begin{array}{l}\text { Solução de modelos } \\
\text { matemáticos utilizando o } \\
\text { método Newton-Rapshon }\end{array}$ & NRTL-E & Cloreto de cálcio \\
\hline Gil et al., 2014 & $\begin{array}{l}\text { Estudo por meio de simulação e } \\
\text { determinação de condições de } \\
\text { operação para a destilação } \\
\text { extrativa de etanol. Análise do } \\
\text { efeito da concentração do agente } \\
\text { extrator. Comparação com } \\
\text { dados experimentais }\end{array}$ & Aspen Plus & NRTL & $\begin{array}{l}\text { Mistura etilenoglicol- } \\
\text { glicerol }\end{array}$ \\
\hline Shang et al., 2019 & $\begin{array}{c}\text { Análise do processo de } \\
\text { destilação extrativa para } \\
\text { separação de uma mistura água- } \\
\text { etanol utilizando um solvente } \\
\text { eutético profundo. Simulação e } \\
\text { otimização do processo. } \\
\text { Comparação com dados } \\
\text { experimentais. }\end{array}$ & $\begin{array}{c}\text { Aspen Plus e Aspen } \\
\text { Dynamics }\end{array}$ & NRTL & $\begin{array}{l}\text { Mistura cloreto de } \\
\text { colina-ureia }\end{array}$ \\
\hline Shi et al., 2020 & $\begin{array}{l}\text { Simulação e otimização de três } \\
\text { configurações de destilação } \\
\text { extrativa para separação de } \\
\text { etanol, terc-butanol e água. } \\
\text { Comparação com dados } \\
\text { experimentais. }\end{array}$ & Aspen Plus & UNIQUAC & Etilenoglicol \\
\hline
\end{tabular}

Fonte: Autores, (2020). 
Figura 3: Representação da coluna de destilação extrativa.

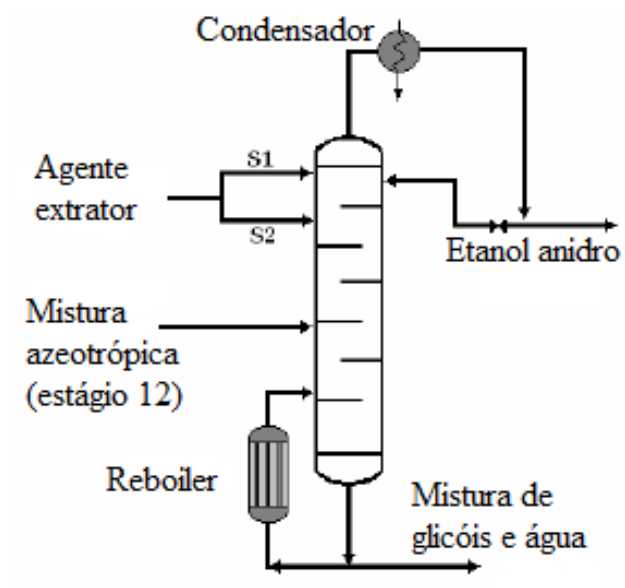

Fonte: Gil et al. (2014).

\section{Metodologia}

A metodologia utilizada no trabalho foi de caráter teórico-computacional, sendo utilizado a versão livre do software computacional ChemSep versão 8.23 para a realização da simulação da destilação extrativa. Como estudo de casos, foi avaliado o sistema de separação de uma mistura água e etanol com a introdução de etilenoglicol como solvente. O cálculo dos coeficientes de atividade para as misturas foi realizado utilizando o modelo termodinâmico UNIFAC (UNIQUAC Functionalgroup Activity Coefficients), que foi validado no software por meio de comparação com dados experimentais de equilíbrio. A metodologia utilizada para desenvolvimento da análise quantitativa para operação de destilação extrativa foi baseada em trabalho previamente desenvolvido (Gil et al., 2014).

O modelo UNIFAC é utilizado na predição de coeficientes de atividade de diversas misturas líquidas não eletrolíticas binárias e multicomponentes. O modelo é derivado para misturas com compostos de tamanho molecular similares, por isso apresenta resultados imprecisos para misturas com componentes de tamanhos distintos. (Muzenda, 2013).

\subsection{Parâmetros iniciais de simulação}

Estudos anteriores realizados por Gil et al. (2014) e Neves et al. (2016) mostraram uma configuração de coluna com 18 a 20 estágios totais, com o solvente sendo alimentado acima da corrente de etanol hidratado. Neste estudo, a simulação foi realizada inicialmente em uma coluna de 18 estágios com alimentação do solvente e da mistura alcoólica nos estágios 4 e 10 respectivamente. A Figura 4 apresenta a configuração da coluna utilizada na simulação.

Os parâmetros iniciais foram determinados com base no estudo realizado por Gil et al. (2014). A corrente de alimentação da mistura azeotrópica foi introduzida com uma vazão de $100 \mathrm{kmol} / \mathrm{h}$, sendo $88 \%$ etanol e $12 \%$ água em base molar. Já para a corrente do agente extrator, a vazão de alimentação introduzida foi de $80 \mathrm{kmol} / \mathrm{h}$. Os demais parâmetros estão dispostos na Tabela 1 . 
Figura 4: Configuração inicial da coluna de destilação extrativa.

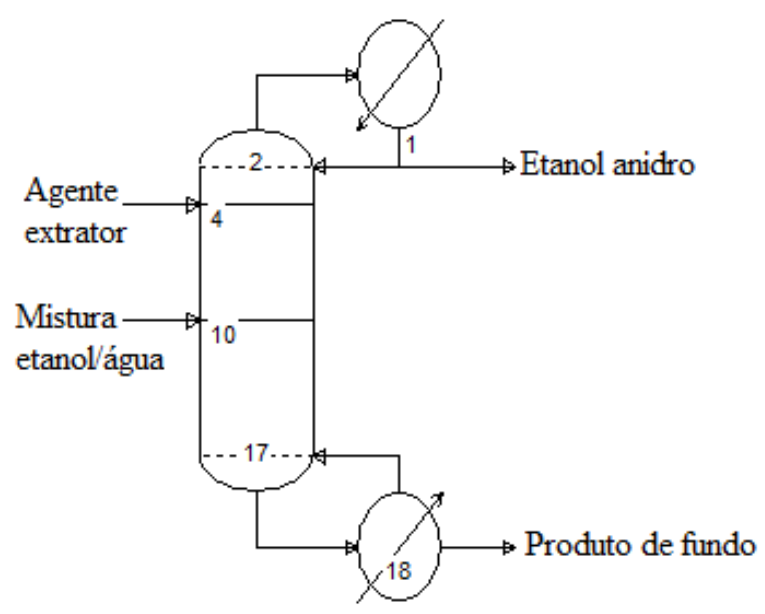

Fonte: Autores (2020).

Tabela 1: Parâmetros iniciais para simulação.

\begin{tabular}{cc}
\hline Parâmetros de simulação & Valor \\
\hline Razão de refluxo & 0,5 \\
Pressão & 1 atm (constante) \\
Temperatura de entrada do solvente & $75^{\circ} \mathrm{C}$ \\
Temperatura de entrada da mistura azeotrópica & $26,85^{\circ} \mathrm{C}$ \\
Taxa molar de produto de fundo & $93,2 \mathrm{kmol} / \mathrm{h}$ \\
\hline
\end{tabular}

Fonte: Gil et al. (2014).

\subsection{Análise de sensibilidade}

A análise de sensibilidade para o processo de destilação extrativa foi efetuada com o auxílio da ferramenta "Parametric Study" do software ChemSep, com objetivo de encontrar as condições de operação mais eficientes da coluna, observando as alterações na concentração de etanol no destilado a partir da variação de parâmetros do processo visando atingir no mínimo $99,5 \%$ de etanol no destilado.

Os parâmetros estudados foram: número total de estágios, estágio de alimentação da mistura azeotrópica e de alimentação do solvente, razão de refluxo e temperatura de entrada do solvente. Estes foram estudados dentro de faixas estipuladas previamente e que estão relatadas na Tabela 2. Para cada valor de maior eficiência obtido na faixa avaliada, este foi considerado fixo na determinação do parâmetro seguinte.

A mesma análise foi realizada para uma eficiência de $100 \%$ e $70 \%$ nos estágios da coluna. A eficiência da coluna é definida como a relação do comportamento de um estágio real com o ideal. Não é possível obter $100 \%$ de eficiência em um processo de destilação real pois diversos fatores podem afetar a transferência de massa entre as fases, como a formação de espuma, velocidade das fases líquida e vapor e o espaçamento entre os pratos da coluna (Belincanta, 2004).

Para colunas de destilação bem dimensionadas, a eficiência dos pratos ocorre entre 70\% e 100\% (Seader et al., 2010). Assim, o presente trabalho investiga ambas as eficiências. Os dados encontrados serão apresentados e discutidos a seguir nos resultados da simulação, junto com gráficos elaborados no software ChemSep para análise das variáveis de processo. 
Research, Society and Development, v. 10, n. 4, e45610414228, 2021

(CC BY 4.0) | ISSN 2525-3409 | DOI: http://dx.doi.org/10.33448/rsd-v10i4.14228

Tabela 2: Faixas estipuladas para a análise de sensibilidade.

\begin{tabular}{cc}
\hline Parâmetros do processo & Faixas de análise \\
\hline Número total de estágios & $11-35$ \\
\hline Estágio de alimentação da mistura azeotrópica & $5-19$ \\
Estágio de alimentação do solvente & $2-10$ \\
Razão de refluxo & $0,3-2,5$ \\
Temperatura de entrada do solvente $\left({ }^{\circ} \mathrm{C}\right)$ & $0-110$ \\
Temperatura de entrada da mistura $\left({ }^{\circ} \mathrm{C}\right)$ & $0-110$ \\
\hline
\end{tabular}

Fonte: Autores (2020).

\section{Resultados e Discussão}

\subsection{Determinação do modelo termodinâmico}

O modelo termodinâmico UNIFAC foi utilizado e validado por meio de comparação com dados de equilíbrio com auxílio da ferramenta " $\Delta$ Data" do software ChemSep, que quantifica o desvio entre os pontos experimentais e a curva de líquido e vapor saturado do diagrama de equilíbrio. A figura 5 mostra o ajuste do modelo para a mistura pseudo-binária etanol/etilenoglicol/água. Na Tabela 3 estão dispostos os desvios obtidos em comparação aos dados experimentais expostos por Li et al. (2000).

Figura 5: Ajuste do modelo UNIFAC aos dados experimentais.

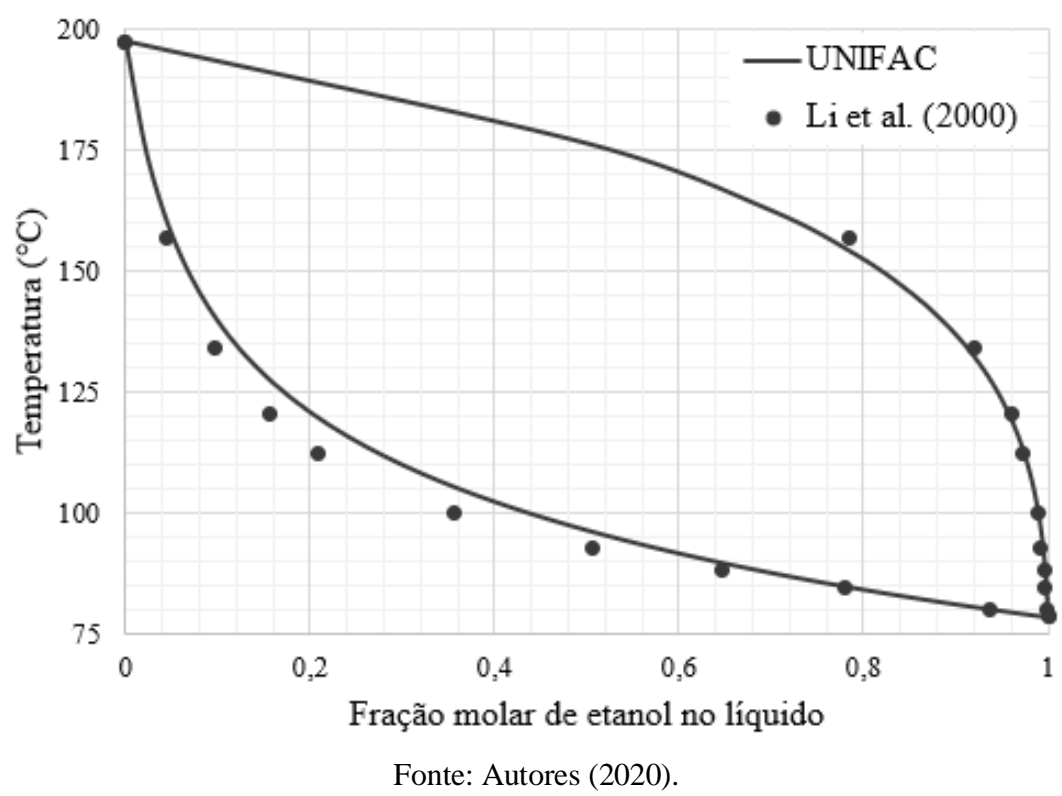

Dias (2016) contatou em seu estudo que um desvio máximo de $6,10 \%$ representa um ajuste adequado do modelo aos dados experimentais. Neste estudo os desvios obtidos foram inferiores $(5,00 \%$ e $0,03 \%)$, desse modo considerou-se o modelo UNIFAC como apropriado para a determinação dos coeficientes de atividade. 
Research, Society and Development, v. 10, n. 4, e45610414228, 2021

(CC BY 4.0) | ISSN 2525-3409 | DOI: http://dx.doi.org/10.33448/rsd-v10i4.14228

Tabela 3: Desvio entre os pontos experimentais e as curvas teóricas.

\begin{tabular}{c|c}
\hline Curvas & Desvio (\%) \\
\hline Líquido saturado & 5,00 \\
Vapor saturado & 0,03 \\
\hline
\end{tabular}

Fonte: Autores (2020).

\subsection{Simulação e análise de sensibilidade}

O primeiro parâmetro analisado foi a quantidade total de estágios na coluna, iniciando a simulação com 18 estágios e variando de 1 a 1 dentro da faixa estipulada na Tabela 2. O ponto de maior eficiência foi determinado através da avaliação da porcentagem molar de etanol no destilado. A Figura 6 mostra o comportamento da curva de recuperação de etanol no destilado com a variação do número total de estágios, do estágio de alimentação da mistura azeotrópica e do solvente.

A Figura 6a indica que inicialmente há um aumento na porcentagem de etanol com o aumento do número total de estágios, e atinge-se a estabilidade. Observou-se que, para 70\% de eficiência, a estabilidade foi atingida com um número maior de estágios devido à necessidade de mais estágios de contato entre as fases para obtenção da concentração mais alta de etanol no destilado. Determinou-se que não há mais variações na porcentagem de etanol após 29 e 20 estágios totais para $70 \%$ e $100 \%$ de eficiência respectivamente, esses foram então fixados para as análises posteriores.

A figura $6 \mathrm{~b}$ mostra o comportamento da porcentagem de etanol no destilado com a variação do estágio de alimentação da mistura azeotrópica, nota-se que há um ponto de máximo na alimentação da mistura no estágio 11 para 100\% de eficiência e no estágio 15 para 70\%, esses foram determinados como de maior eficiência e fixados para estudo dos demais parâmetros.

O mesmo estudo foi realizado com a alimentação do solvente na coluna, observando-se na figura 6c que se alcançou o ponto de maior eficiência com o solvente sendo introduzido no estágio 3 e no estágio 5 para $100 \%$ e $70 \%$ de eficiência respectivamente.

A Figura 7 mostra a análise para a razão de refluxo, a temperatura de entrada do solvente e da mistura azeotrópica. A razão de refluxo é a relação entre a quantidade de líquido que retorna para coluna como refluxo e o que é retirado como produto de topo, o seu aumento acarreta maior consumo de energia do condensador e do refervedor (Oliveira et al., 2016). A Figura 7a mostra um crescimento acentuado da porcentagem molar de etanol com o aumento da razão de refluxo até atingir a estabilidade. Como a concentração requerida de etanol (99,5\% molar) já é atingida com os valores de 1,1 (100\% de eficiência) e 1,4 (70\% de eficiência), esses foram determinados como pontos de operação buscando uma economia de energia, uma vez que maiores valores gerariam gastos desnecessários para o condensador e para o refervedor. 
Figura 6: Determinação das condições de maior eficiência: (a) número total de estágios (b) estágio de alimentação d mistura azeotrópica (c) estágio de alimentação do solvente.

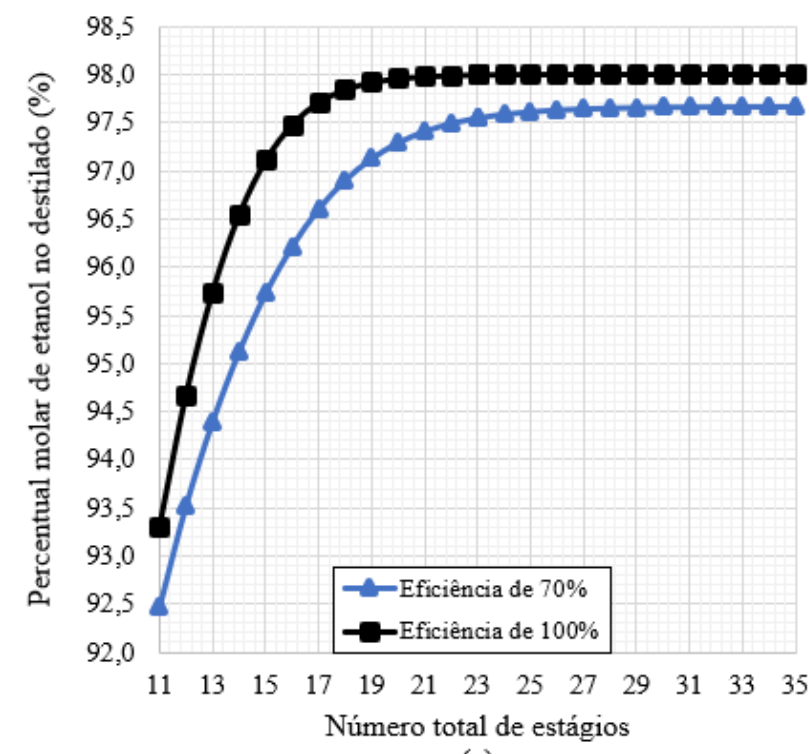

(a)

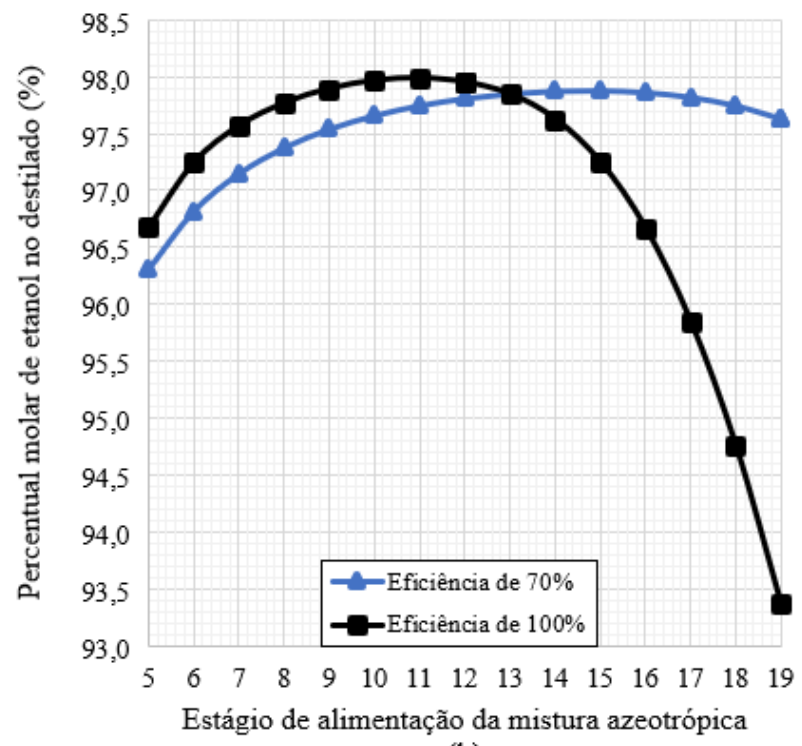

(b)

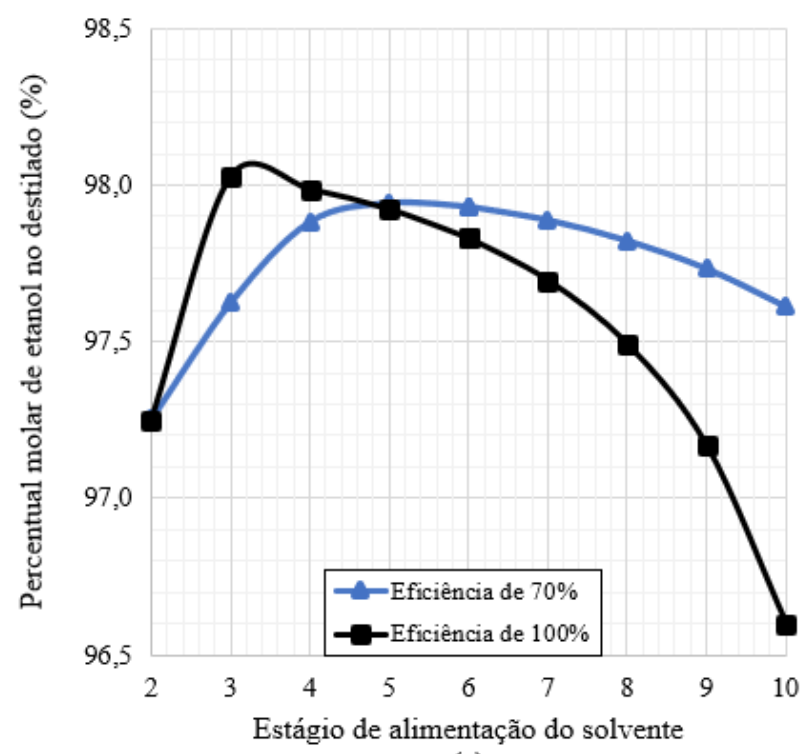

(c)

Fonte: Autores (2020).

Analisando a Figura 7b, pode-se perceber que a redução na temperatura de alimentação do solvente gerou um destilado com pureza maior de etanol. Como a concentração desejada de etanol já foi atingida, a redução na temperatura de entrada de solvente não é necessária. Considerou-se a temperatura de $70^{\circ} \mathrm{C}$ como adequada em ambas as eficiências.

A Figura 7c demonstra que não houve grandes alterações no percentual de álcool com a variação da temperatura de alimentação da mistura azeotrópica na faixa de 0 a $75^{\circ} \mathrm{C}$. Em temperaturas superiores, a alimentação será uma mistura vaporlíquido, sendo possível verificar uma queda na concentração de etanol no destilado, cujo fato é esperado, uma vez que a água é arrastada com o fluxo de vapor ascendente (Gil, 2014). Segundo Oliveira et al. (2016), temperaturas baixas podem gerar quedas de pressão indesejadas no interior da coluna. Devido a esses motivos, determinou-se a temperatura de $25^{\circ} \mathrm{C}$ como adequada, na faixa estudada para ambas as eficiências. 
Research, Society and Development, v. 10, n. 4, e45610414228, 2021

(CC BY 4.0) | ISSN 2525-3409 | DOI: http://dx.doi.org/10.33448/rsd-v10i4.14228

Figura 7: Determinação das condições de maior eficiência: (a) razão de refluxo (b) temperatura de alimentação do solvente (c) :emperatura de alimentação da mistura.

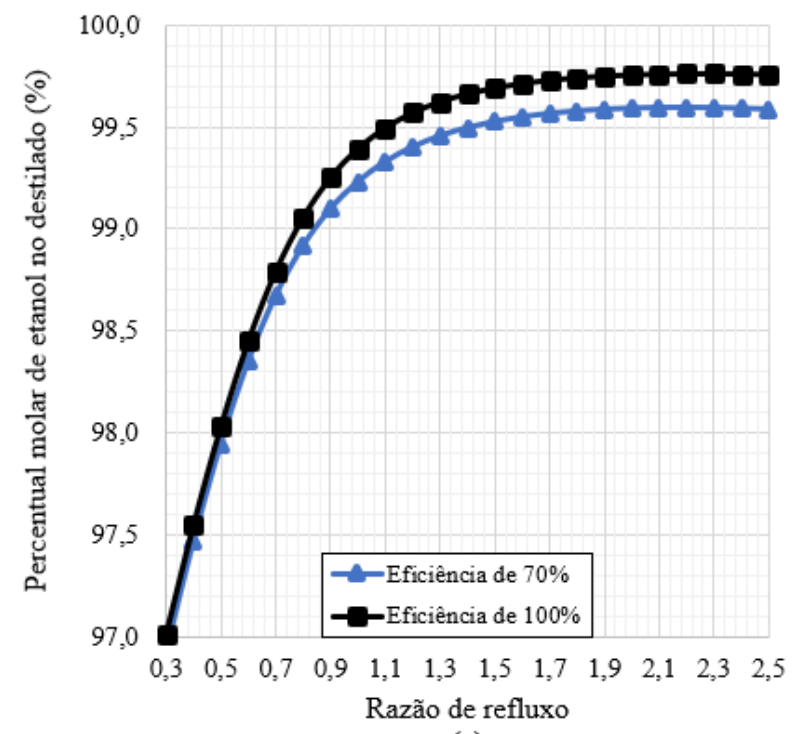

(a)

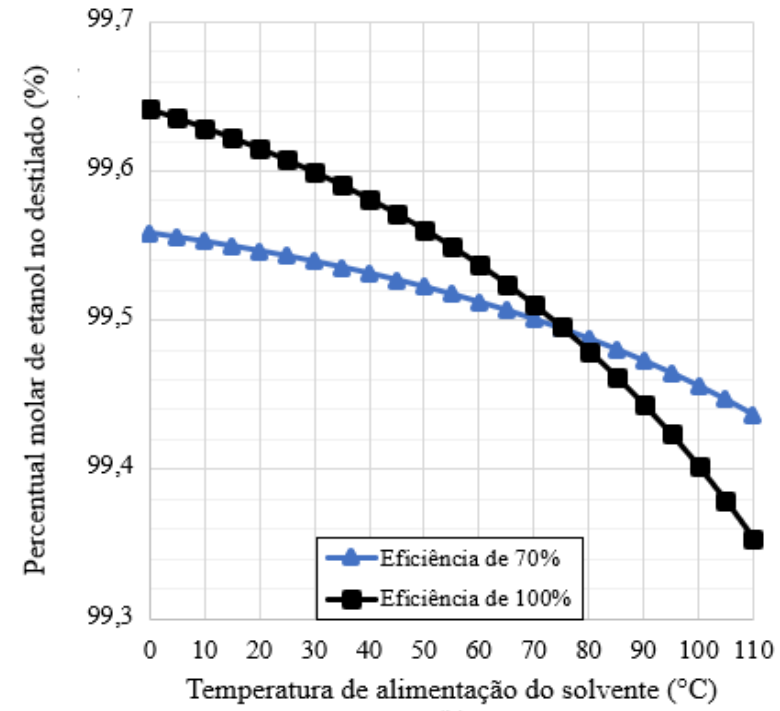

(b)

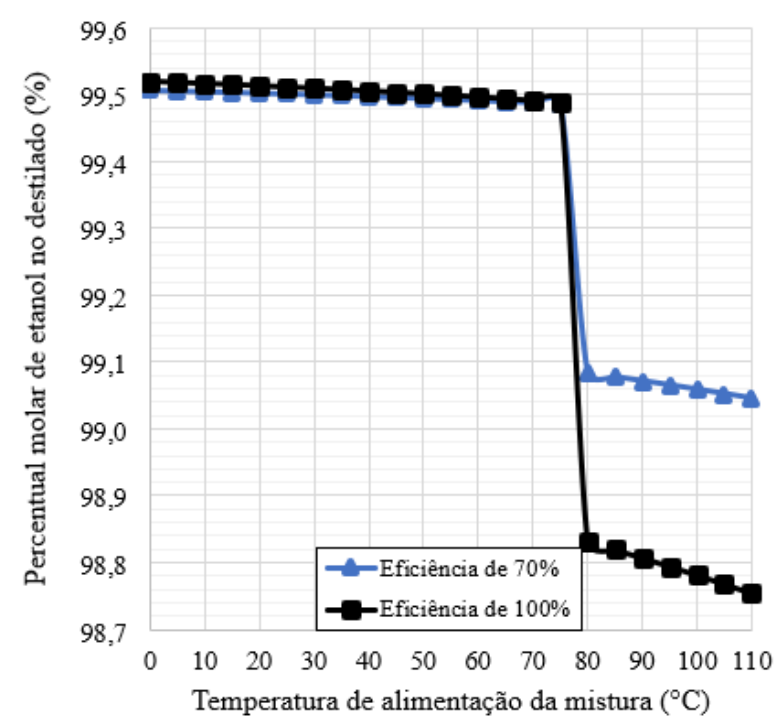

(c)

Fonte: Autores (2020).

A Tabela 4 apresenta o dimensionamento da coluna com as condições de operação adotadas para as eficiências estudadas, junto com as estimativas de percentual de etanol e de taxa molar de destilado obtidas após a análise.

É possível verificar na Tabela 4 que, após a análise de sensibilidade para obtenção da coluna com configuração de maior eficiência para o processo, o percentual mínimo de etanol para produção de etanol anidro foi atingido com a utilização do etilenoglicol como agente extrator. É possível verificar a necessidade de uma quantidade maior de estágios e um valor maior de razão de refluxo considerando uma coluna com $70 \%$ de eficiência dos estágios, o que significa também um maior consumo de energia, além de maior custo fixo. 
Tabela 4: Dimensionamento da coluna e estimativa de percentual de etanol.

\begin{tabular}{ccc}
\hline Parâmetro & $\mathbf{1 0 0 \%}$ de eficiência & 70\% de eficiência \\
\hline Número total de estágios & 20 & 29 \\
\hline Estágio de alimentação da mistura azeotrópica & 11 & 15 \\
Estágio de alimentação do solvente & 3 & 5 \\
Razão de refluxo & 1,1 & 1,4 \\
Temperatura de alimentação do solvente $\left({ }^{\circ} \mathrm{C}\right)$ & 70 & 70 \\
Temperatura de alimentação da mistura $\left({ }^{\circ} \mathrm{C}\right)$ & 25 & 25 \\
Percentual molar de etanol no destilado $(\%)$ & 99,51 & 99,50 \\
Taxa molar de etanol no destilado $(\mathrm{kmol} / \mathrm{h})$ & 86,80 & 86,80 \\
\hline
\end{tabular}

Fonte: Autores (2020).

Gil et al. (2014) obteve resultados similares na simulação do processo de destilação extrativa com 100\% de eficiência dos estágios: uma coluna com 20 estágios totais, com alimentação da mistura azeotrópica no estágio 12 na temperatura de $20^{\circ} \mathrm{C}$ e o solvente no estágio 4 na temperatura de $70^{\circ} \mathrm{C}$ com razão de refluxo de 0,92 . Figueirêdo (2009) realizou um estudo do comportamento da fração molar de etanol no destilado com a variação da razão de refluxo, observou-se um comportamento semelhante ao obtido neste trabalho, um crescimento acentuado seguido de estabilização.

Neste estudo, destaca-se que a pressão no interior da coluna foi considerada constante, fato que não ocorre em uma coluna de destilação real, pois uma diferença de pressão entre a base e o topo é necessária para evitar gotejamentos, inundações e quedas de pressão em estágios superiores (Oliveira, et al., 2016).

\section{Conclusão}

A simulação utilizando o software computacional livre ChemSep se mostrou eficiente na predição de respostas do processo de destilação. O modelo termodinâmico UNIFAC foi utilizado e se ajustou adequadamente aos dados experimentais para a mistura pseudo-binária etanol/etilenoglicol/água.

Verificou-se que na simulação o percentual molar requerido de etanol na corrente de topo da coluna (99,5\%) foi atingido após a análise de sensibilidade e obtenção dos parâmetros mais eficientes. Em relação aos parâmetros de processo, para uma coluna com $100 \%$ de eficiência, determinou-se a alimentação da mistura azeotrópica no estágio 11 e do solvente no estágio 5 em uma coluna de 20 estágios totais como a configuração mais eficiente na faixa estudada. As temperaturas de 70 e $40^{\circ} \mathrm{C}$ foram definidas para a alimentação do solvente e da mistura etanol/água respectivamente, para uma razão de refluxo de 1,1 .

Entretanto, sabe-se que em um processo real, há vários fatores que afetam a eficiência da transferência de massa entre as fases, devido a isso realizou-se a análise com $70 \%$ de eficiência buscando uma maior aproximação do processo real. Comparando os resultados obtidos para eficiência menor, será necessário um número maior de estágios (29 estágios totais), com o solvente sendo introduzido no estágio 5 e a mistura azeotrópica no estágio 15 , e uma maior razão de refluxo $(1,4)$ para obtenção de mesma concentração de etanol e consequentemente um maior gasto de energia.

Algumas sugestões para trabalhos futuros são realizar novas simulações alterando as pressões do topo e da base da coluna, buscando obter parâmetros ainda mais aproximados do processo real. Além disso, sugere-se realizar a análise econômica, considerando os valores de custos fixo e operacional. 
Research, Society and Development, v. 10, n. 4, e45610414228, 2021

(CC BY 4.0) | ISSN 2525-3409 | DOI: http://dx.doi.org/10.33448/rsd-v10i4.14228

\section{Referências}

Abdellatief, T. M. M., Ershov, M. A., Kapustin, V. M., Abdelkareem, M. A., Kamil, M. \& Olabi, A. G. (2021). Recent trends for introducing promising fuel components to enhance the anti-knock quality of gasoline: A systematic review. Fuel 291, (120112), 1-24. 10.1016/j.fuel.2020.120112

Belincanta, J. (2004). Coluna de para-destilação: análise das características hidrodinâmicas e de eficiência de Murphree. Dissertação de mestrado, Faculdade de Engenharia Química. Universidade Estadual de Campinas, Campinas.

Bessa, L. C. B. A. (2012). Integração térmica de colunas de destilação de destilação alcoólica multicomponente. Dissertação de mestrado, Faculdade de Engenharia de alimentos. Universidade Estadual de Campinas, Campinas.

Cavalcanti, C. J. S. (2018). Simulação e otimização da produção de etanol anidro combustível. Dissertação de mestrado. Programa de Pós-Graduação em Engenharia Química. Universidade Federal de Pernambuco, Recife.

CONAB. Companhia Nacional de Abastecimento. (2020). https://www.conab.gov.br/info-agro/safras/cana.

Dias, M. O. S., Maciel Filho, R., Mantelatto, P. E., Cavallet, O., Rosell C. E. V., Bonomi, A. \& Leal, M. R. L. V. (2015). Sugarcane processing for ethanol and sugar in Brazil. Environmental Development, 15, 35-51. 10.1016/j.envdev.2015.03.004.

Dias, R. M. (2016). Estudo de equilíbrio líquido-vapor de soluções hidroetanólicas contendo frutose. Dissertação de mestrado. Programa de Pós-Graduação em Engenharia Química. Universidade Federal de São Carlos, São Carlos.

Figueirêdo, M. F. (2009). Obtenção de etanol anidro via destilação extrativa: Simulação e otimização. Dissertação de mestrado. Faculdade de Engenharia Química. Universidade Federal de Campina Grande, Campina Grande.

Gil, I. D, Gárcia, L. C \& Rodríguez, G. (2014). Simulation of ethanol extractive distillation with mixed glycols as separating agent. Brazilian Journal of Chemical Engineering, 31(01), 259-270. 10.1590/S0104-66322014000100024.

Gimenez, A. R., Altopiedi, L. G., Carballo, N. V., Silva, L. C. M. \& Liria, C. W. (2018). O aumento da produtividade e a busca pela excelência na produção do etanol brasileiro: uma história de sucesso. Research, Society and Development, 7(2), 01-19. 10.17648/rsd-v7i2.270.

Leal, M. R. L. V. (2014). Technological evolution of sugarcane processing for ethanol and electric power generation. Sugarcane bioethanol R\&D for Productivity and Sustainability, 561-576.

Li, J., Chen, C. \& Wang, J. (2000). Vapor-liquid equilibrium data and their correlation for binary systems consisting of ethanol, 2-propanol, 1,2-ethanediol and methyl benzoate. Fluid Phase Equilibria, 169, 75-84.

Lopes, C. H, Gabriel A. V. M. D \& Borges, M. T. M. R. (2011). Produção de etanol a partir da cana-de-açúcar. Universidade Federal de São Carlos. http://audiovisual.uab.ufscar.br/impresso/2016/TS/TS_Claudio_ProducaoEtanol.pdf.

Muller, T. A., Resende, J. T. V., Spinosa, W. A., Guerra, E. P. \& Constantino, L. V. (2019). Produção de álcool a partir de cultivares de batata nas seleções especial e refugo. Research, Society and Development, 9(2), 1-22. 10.33448/rsd-v9i2.192.

Muzenda, E. (2013). From UNIQUAC to Modified UNIFAC Dortmund: A Discussion. 3rd International Conference on Medical Sciences and Chemical Engineering, Bangkok, Thailand.

Neves, T. G., Villar, S. B. B. L., Freitas, H. F. S. \& Brito, R. P. (2016). Obtenção de bioetanol anidro via destilação extrativa utilizando glicerol e etilenoglicol como solventes. COBEQ, XXI Congresso Brasileiro de Engenharia Química, Fortaleza.

Oliveira, M. A. D., Barbosa, N. A. \& Giati, T. M. (2016). Simulação da obtenção de álcool isoamílico a partir do óleo fúsel via destilação. Trabalho de conclusão de curso. Faculdade de engenharia química. Universidade de São Francisco, Campinas.

PORTARIA No 75, de 5 de março de 2015 do Ministério da Agricultura, Pecuária e Abastecimento. Diário Oficial da União: Seção 1 No 44 (2015). https://pesquisa.in.gov.br/imprensa/jsp/visualiza/index.jsp?jornal=1\&pagina=17\&data=06/03/2015.

Restrepo, M. L. \& Aguilar-Arias, J. (2003). Modeling and simulation of saline extractive distillation columns for the production of absolute ethanol. Computers and Chemical Engineering, 27, 527-549.

Santos, L. S. (2016). Projeto de colunas de destilação sujeito a incertezas. Trabalho de conclusão de curso. Escola de Química e Alimentos. Universidade Federal do Rio Grande, Santo Antônio da Patrulha.

Seader, J. D., Ernest, J. \& Keith, D. (2010). Separation process principles: chemical and biochemical operations. Separation (Technology)-Textbooks.

Shang, X., Ma, S., Pan, Q., Li, J., Sun, Y., Ji, K. \& Sun, L. (2019). Process analysis of extractive distillation for the separation of ethanol-water using deep eutectic solvent as entrainer. Chemical Engineering Research and Design, 148, 298-311. 10.1016/j.cherd.2019.06.014.

Shi, X., Zhu, X., Zhao, X. \& Zhang, Z. (2020). Performance evaluation of different extractive distillation processes for separating ethanol/tert-butanol/water mixture. Process Safety and Environmental Protection, 137, 246-260. 10.1016/j.psep.2020.02.015.

Silva, F. S. G., Gomes, W. P. C. \& Nascimento, D. D. (2019). Avaliação da fermentação de leveduras visando produção de etanol de segunda geração. Bioenergia em revista: diálogos, 9(2), 35-61.

Tadini, C. C., Telis, V. R. N., Meirelles, A. J. A. \& Pessoa Filho, P. A. (2016). Operações Unitárias na Indústria de Alimentos LTC Editora. 